\title{
Detection and Genotyping of Human Papillomavirus DNA in Formalin- Fixed Paraffin-Embedded Specimens with the HPV Direct Flow CHIP System
}

\author{
Elsa Herraez-Hernandez ${ }^{1}$, Ovidiu Preda ${ }^{1}$, Sonia Alonso ${ }^{2}$, Rosario Serrano Pardo ${ }^{3}$ and \\ Asuncion Olmo ${ }^{*}, 1$
}

${ }^{1} R \& D$ Department, Master Diagnóstica, Avenida del Conocimiento 100, Parque Tecnológico de Ciencias de la Salud, 18016, Granada, Spain

${ }^{2}$ Pathology Department, Elda General Hospital. Carretera Elda-Sax, La Torreta s/n, 03600, Elda, Alicante, Spain

${ }^{3}$ Pathology Department, Ruber Clinic, Calle de Juan Bravo, 49, 28006, Madrid, Spain

\begin{abstract}
The novel HPV Direct Flow CHIP commercial system for Human Papillomavirus (HPV) genotyping is based on rapid PCR and automatic reverse dot blot hybridization to genotype-specific probes, allowing the detection of $36 \mathrm{HPV}$ genotypes. This study examined the performance of HPV Direct Flow CHIP in formalin-fixed paraffin-embedded (FFPE) samples $(n=99)$. Each sample was analyzed both by Direct PCR, using crude cell extracts without DNA purification, and by conventional PCR, using purified DNA. Pair-wise analysis of the results demonstrated strong concordance between the results obtained with the two protocols, although a slightly higher rate of multiple infections was detected by conventional PCR. In summary, HPV Direct Flow CHIP achieves effective HPV detection from FFPE samples with both Direct PCR and Conventional PCR protocols.
\end{abstract}

Keywords: Biopsy, cervical cancer, direct PCR, FFPE, HPV, HPV direct flow CHIP.

\section{INTRODUCTION}

Persistent Human Papillomavirus (HPV) infection is the causative agent of benign and malignant squamous epithelial lesions that can lead to cervical cancer [1], and HPV 16 and 18 are responsible for $70 \%$ of cases of this cancer worldwide [2]. With the implementation of prophylactic vaccines against HPV 16 and 18 (Cervarix ${ }^{\circledR}$ - GlaxoSmithKline, Rixensart, Belgium) or HPV 6, 11, 16, and 18 (Gardasil $^{\circledR}{ }_{-}$ Sanofi Pasteur MSD, Lyon, France), a major reduction in the incidence of cervical cancer is expected. Consequently, effective protection against HPV 16 and 18 and possible cross-protection against other genotypes will modify the prevalence of HPV genotypes in vaccinated populations [3, 4]. However, HPV testing will still be required to follow-up lesions caused by genotypes not included in the vaccines and to study their involvement in cervical lesions. HPV genotyping also remains necessary for the adequate screening of non-vaccinated populations and in epidemiologic studies.

HPV testing is mainly performed using dry swabs, liquidbased cytologies, or formalin-fixed paraffin-embedded (FFPE) biopsies as biological material. FFPE specimens are taken from selected target regions of the cervical epithelium, allowing detected HPV genotypes to be linked to specific

*Address correspondence to this author at the R\&D Department, Master Diagnóstica, Avenida del Conocimiento 100, Parque Tecnológico de Ciencias de la Salud, 18016, Granada, Spain; Tel: +34 958271 449; Fax: +34958 271 434; E-mail: asuncion.olmo@vitroweb.com lesions. Hence, sensitive viral detection in FFPE biopsies is important for the screening of cervical cancer and precursor lesions and for epidemiology and vaccinology studies [5]. However, HPV testing is known to perform poorly in FFPE samples due to partial DNA fragmentation in the embedded tissue [6], resulting in lower HPV detection rates and impairing the identification of multiple infections in comparison to fresh cervical smears.

The HPV Direct Flow CHIP test (Master Diagnóstica, Granada, Spain) was recently launched onto the market as a new system for rapid and sensitive HPV genotyping. This PCR-based method is able to identify $36 \mathrm{HPV}$ genotypes: 6, $11,16,18,26,31,33,35,39,40,42,43,44 / 55,45,51,52$, $53,54,56,58,59,61,62,66,67,68,69,70,71,72,73,81$, 82,84 , and 89 . The specific formulation of the PCR mix and the polymerase in the kit allow the PCR to be completed within $60 \mathrm{~min}$. It includes the amplification of a $268 \mathrm{bp}$ fragment of the human beta-globin gene (internal control) and a 150 bp fragment of the HPV L1 region (GP5+/GP6+). The reverse dot blot hybridization and read-out of the results are then performed automatically in the e-BRID System ${ }^{\mathrm{TM}}$ (Master Diagnóstica, Granada, Spain), which takes 90 min to complete a set of 15 samples. Although purified DNA can be the input material for the amplification, this system can also perform so-called Direct PCR by using crude cell extracts from cervical swabs, liquid-based cytologies, or FFPE sections as amplification templates, precluding the need for previous DNA purification. 
The analytical sensitivity and specificity of the HPV Direct Flow CHIP system were certified after enrolment in the 2011 WHO Proficiency Panel (manuscript in preparation); the results of the quality program demonstrated that the kit can specifically detect 5 IU of HPV 16 and 18 and 50 GE of HPV 6, 11, 31, 33, 35, 39, 45, 51, 52, 58, 59, $66,68 \mathrm{a}$ and $68 \mathrm{~b}$ in single and multiple infection settings. The performance of HPV Direct Flow CHIP in cytological samples was found to be highly similar to that of the Linear Array Genotyping Test (Roche Molecular Systems, Alameda, CA, USA), Hybrid Capture 2 (Digene Corp., Gaithersburg, MD, USA), and CLART HPV2 (Genomica, Tres Cantos, Madrid, Spain) commercial assays, by Direct PCR (using crude cell extracts) [7]. In addition, a high agreement was observed among the results of HPV Direct Flow CHIP, Linear Array Genotyping Test, and Direct Sequencing when purified DNA was used as amplification template (submitted for publication). However, the efficacy of HPV detection in purified DNA from FFPE samples was found to be significantly higher with HPV Direct Flow CHIP than with CLART HPV2 in a small pilot study [7].

In order to further characterize the HPV Direct Flow CHIP system, the present study evaluated its performance in cervical FFPE biopsies. Paired samples for Direct PCR and Conventional PCR from FFPE samples were used for the HPV Direct Flow CHIP testing, and the results of the two protocols were compared. Samples $(n=99)$ were collected from patients with an abnormal cytology or under follow-up for cervical lesion between 2010 and 2013 in the Elda General Hospital (Alicante, Spain) and Ruber Clinic (Madrid, Spain). The patients signed their informed consent for all specimens, and the study procedures were approved by the clinical research ethics committees of the hospitals.

The biopsies were fixed for $48 \mathrm{~h}$ in formalin and embedded in paraffin, and FFPE samples were then sectioned. Several serial sections were taken from each biopsy, the two outer sections were stained with hematoxylin-eosin, examined under the microscope, and classified as: normal, mild dysplasia/cervical intraepithelial neoplasia (CIN) grade 1 (CIN1), moderate dysplasia/CIN grade 2 (CIN2), severe dysplasia/CIN grade 3 (CIN3), or cervical carcinoma. The inner sections were used for HPV detection by using the HPV Direct Flow CHIP with two different protocols (Direct and Conventional PCR). For the Direct PCR, one to three sections were digested in $60 \mu \mathrm{L}$ Lysis Buffer (Master Diagnóstica, Granada, Spain) and 1.5 $\mu \mathrm{L}$ of DNA Release (Master Diagnóstica, Granada, Spain) for $30 \mathrm{~min}$ at $60^{\circ} \mathrm{C}$ in a MJ Mini ${ }^{\mathrm{TM}}$ Thermal Cycler (BioRad, Hercules, CA, USA), followed by inactivation at $98^{\circ} \mathrm{C}$ for $10 \mathrm{~min}$. Six microliters of the extracts obtained were added to $53 \mu \mathrm{L}$ of the PCR mix and $1 \mu \mathrm{L}$ of DNA polymerase supplied in the kit (Master Diagnóstica, Granada, Spain). For the Conventional PCR, the purified DNA to be used as amplification template was automatically extracted from other one to three inner sections, using the Maxwell $^{\circledR} 16$ system with Maxwell ${ }^{\circledR} 16$ FFPE Plus Lev DNA Purification Kit (Promega, Madison, Wisconsin, USA), and eluted in 30-50 microliters of TE buffer following the manufacturer's instructions. Then, $6 \mu \mathrm{L}$ of the corresponding template were added to $53 \mu \mathrm{L}$ of PCR mix and $1 \mu \mathrm{L}$ of DNA polymerase. The cycling conditions in the
MJ Mini ${ }^{\mathrm{TM}}$ Thermal Cycler, identical for both protocols, were: denaturation at $98^{\circ} \mathrm{C}$ for $5 \mathrm{~min}$; 5 cycles of denaturation at $98^{\circ} \mathrm{C}$ for $5 \mathrm{~s}$, annealing at $42^{\circ} \mathrm{C}$ for $5 \mathrm{~s}$, and elongation at $72^{\circ} \mathrm{C}$ for $10 \mathrm{~s} ; 45$ cycles of denaturation at $98^{\circ} \mathrm{C}$ for $5 \mathrm{~s}$, annealing at $60^{\circ} \mathrm{C}$ for $5 \mathrm{~s}$, and elongation at $72^{\circ} \mathrm{C}$ for $10 \mathrm{~s}$; and final elongation at $72^{\circ} \mathrm{C}$ for $1 \mathrm{~min}$. Amplicons were denatured for $5 \mathrm{~min}$ at $95^{\circ} \mathrm{C}$ and hybridized in the e-BRID System ${ }^{\mathrm{TM}}$ (Master Diagnóstica, Granada, Spain) as previously described [7]. The results obtained with the two protocols were compared, obtaining the overall agreement, positive agreement, and Cohen's Kappa coefficient and applying the McNemar chi-square test.

The study population $(n=99$, mean age 33.86 yrs, standard deviation of 9.7 yrs) was histologically distributed as follows: $15.2 \%$ normal $(n=15), 52.5 \%$ CIN1 $(n=52)$, $20.2 \%$ CIN2 $(n=20), 7.1 \%$ CIN3 $(n=7)$, and $5.1 \%$ carcinoma $(n=5)$. All samples tested were positive for the human betaglobin internal control in both protocols and were therefore considered valid. The agreement between the two methods for overall HPV positivity was $98.98 \%$ (Direct PCR: 93 samples HPV-positive and 5 HPV-negative; Conventional PCR: 94 samples HPV-positive and 4 HPV-negative). The overall positive agreement between the methods was very high (Table 1), and although a slightly higher rate of HPV detection was observed with Conventional PCR, the difference was not statistically significant. Likewise, as shown in Table 2 , no significant difference was found in the detection of multiple-infection cases (Direct PCR: 66 cases, Conventional PCR: 71 cases; Kappa index $=0.49)$. Study of the genotype-specific agreement indicated a high concordance between the protocols in the detection of carcinogenic genotypes, with Kappa indexes ranging from moderate to perfect (Table 3 ). The most prevalent genotypes were HPV 16, 6, and 18, although their prevalence varied according to the lesion grade, ranging from $58.7 \%, 28.6 \%$ and $12.7 \%$ (Direct PCR) or $54 \%, 33.3 \%$ and $11.1 \%$ (Conventional PCR), respectively, in <CIN2 patients, to $65.6 \%, 15.6 \%$ and $25 \%$ (Direct PCR) or $56.3 \%, 25 \%$ and $28.1 \%$ (Conventional PCR) in $\geq$ CIN2 patients. Slightly higher detection rates were obtained with Direct (versus Conventional) PCR for HPV 16, 43 and 52 and with Conventional (versus Direct) PCR for HPV 6, 11 and 61. The small non-significant differences between the two protocols may be attributable to a lower amplification yield for the non-purified templates, probably due to the presence of PCR inhibitors from cell lysates in the Direct PCR mixture. However, the agreement between the protocols was very good (Table 1), especially in $\geq \mathrm{CIN} 2$ patients $(100 \%)$, suggesting that the results are more similar with greater disease severity, as previously observed for the HPV Direct Flow CHIP system [7]. Analysis of the clinical performance of HPV Direct Flow CHIP showed the same sensitivity value (100\%, CI 95\%; 89.28-100) and negative predictive value $(100 \%)$ for both protocols. The positive predictive value was $34.04 \%$ for Direct PCR and 33.68\% for Conventional PCR.

Unlike other systems, such as the Linear Array Genotyping Test, in which the internal control amplified fragment is smaller than the HPV amplicon (268 bp versus $450 \mathrm{bp}$ ), the HPV Direct Flow CHIP test is based on the amplification of a beta-globin control fragment of $268 \mathrm{bp}$ and an HPV fragment of approximately $150 \mathrm{bp}$. Given that 
Table 1. Overall HPV Detection by HPV Direct Flow CHIP Using Direct PCR and Conventional PCR Protocols

\begin{tabular}{|c|c|c|c|c|c|c|}
\hline & Direct PCR/ Conventional PCR & N of Samples & Agreement & Positive Agreement & $\begin{array}{c}\text { Kappa Index } \\
\text { (95\% CI) }\end{array}$ & McNemar p-Value \\
\hline \multirow{6}{*}{$<\mathrm{CIN} 2(\mathrm{~N}=67)$} & Positive/positive & 62 & \multirow{6}{*}{98.51} & \multirow{6}{*}{92.54} & \multirow{6}{*}{$0.88(0.65-1)$} & \multirow{6}{*}{1} \\
\hline & Concordant & 23 & & & & \\
\hline & Compatible & 39 & & & & \\
\hline & Positive/negative & 0 & & & & \\
\hline & Negative/positive & 1 & & & & \\
\hline & Negative/negative & 4 & & & & \\
\hline \multirow{6}{*}{$\geq \mathrm{CIN} 2(\mathrm{~N}=32)$} & Positive/positive & 32 & \multirow{6}{*}{100} & \multirow{6}{*}{100} & \multirow{6}{*}{-} & \multirow{6}{*}{-} \\
\hline & Concordant & 15 & & & & \\
\hline & Compatible & 17 & & & & \\
\hline & Positive/negative & 0 & & & & \\
\hline & Negative/positive & 0 & & & & \\
\hline & Negative/negative & 0 & & & & \\
\hline
\end{tabular}

Concordant $=$ exactly the same genotype $(\mathrm{s})$ detected by both methods; Compatible $=$ at least one genotype detected by both methods. $\mathrm{N}=$ number . $\mathrm{CI}=$ confidence interval.

Table 2. Detection of Multiple Infections by HPV Direct Flow CHIP Using Direct PCR and Conventional PCR Protocols

\begin{tabular}{|c|c|c|c|c|c|c|c|}
\hline \multirow{2}{*}{ Genotypes Detected by Conventional PCR (N) } & \multicolumn{5}{|c|}{ Genotypes Detected by Direct PCR (N) } & $\begin{array}{c}\text { Kappa } \\
\text { Index }(95 \% \text { CI })\end{array}$ & $\begin{array}{l}\text { Mc Nemar } \\
\text { p-Value }\end{array}$ \\
\hline & None & One & Two & Three & Four or More & \multirow{6}{*}{$0.49(0.37-0.62)$} & \multirow{6}{*}{0.289} \\
\hline None & 4 & 0 & 0 & 0 & 0 & & \\
\hline One & 1 & 17 & 4 & 1 & 0 & & \\
\hline Two & 0 & 11 & 13 & 6 & 2 & & \\
\hline Three & 0 & 0 & 5 & 10 & 1 & & \\
\hline Four or more & 0 & 0 & 6 & 1 & 17 & & \\
\hline
\end{tabular}

$\mathrm{N}$ : number. CI: confidence interval.

the DNA tends to be partially fragmented in FFPE samples, the correct amplification of this internal control fragment demonstrates the viability of the sample to amplify a $150 \mathrm{bp}$ fragment of the HPV genome with the HPV Direct Flow CHIP system. This PCR formulation reduces the likelihood of a false negative result, because the HPV will be amplified more efficiently than the beta-globin fragment; therefore, a positive signal for beta-globin and negative signal for HPV should indicate a true HPV-negative sample. Besides the design of the PCR mix, the high sensitivity of the test in FFPE samples can be attributed to the small size of the HPV amplicons. Differences in sensitivity rates as a function of amplicon size were previously reported for other systems based on the amplification of small viral sequences. Thus, the INNO-LiPA assay (Innogenetics, Ghent, Belgium) which includes SPF10 primers targeting a small viral region of $65 \mathrm{bp}$, proved less prone to degradation and more sensitive to detect HPV in FFPE samples in comparison to systems with larger amplicon sizes, such as HPV2 CLART (HPV amplicon size: $450 \mathrm{bp}$ ) [8, 9] and Linear Array Genotyping Test (HPV amplicon size: $450 \mathrm{bp}$ ) [10]. Further research is warranted to compare the performance of the HPV Direct Flow CHIP in FFPE samples with that of INNO-LiPA and other highly sensitive techniques.

Finally, the use of different commercial kits for DNA purification can produce changes in the rates of HPV detection due to DNA degradation and cross-linkage, leading to low intra-assay reproducibility, as reported for the Linear Array Genotyping Test and INNO-LiPA methods [11]. In the present study, the sensitivity of the HPV Direct Flow CHIP system did not vary between the use of DNA purified by means of the Maxwell ${ }^{\circledR} 16$ FFPE Plus Lev DNA Purification Kit and the use of non-purified material.

In conclusion, pair-wise comparisons demonstrated a high agreement between Direct PCR and Conventional PCR protocols in the detection of HPV in FFPE samples with the HPV Direct Flow CHIP, which demonstrated a very high sensitivity in this type of sample. A larger number of genotypes were detected in multiple-infection by Conventional versus Direct PCR, but the clinical sensitivity and specificity values were similar between the two approaches. HPV detection and genotyping is crucial in FFPE specimens, especially in the follow-up of patients with 
Table 3. Comparison of Genotype Detection by Direct PCR and Conventional PCR: Overall Agreement, Positive Agreement, Kappa Index, and McNemar Test

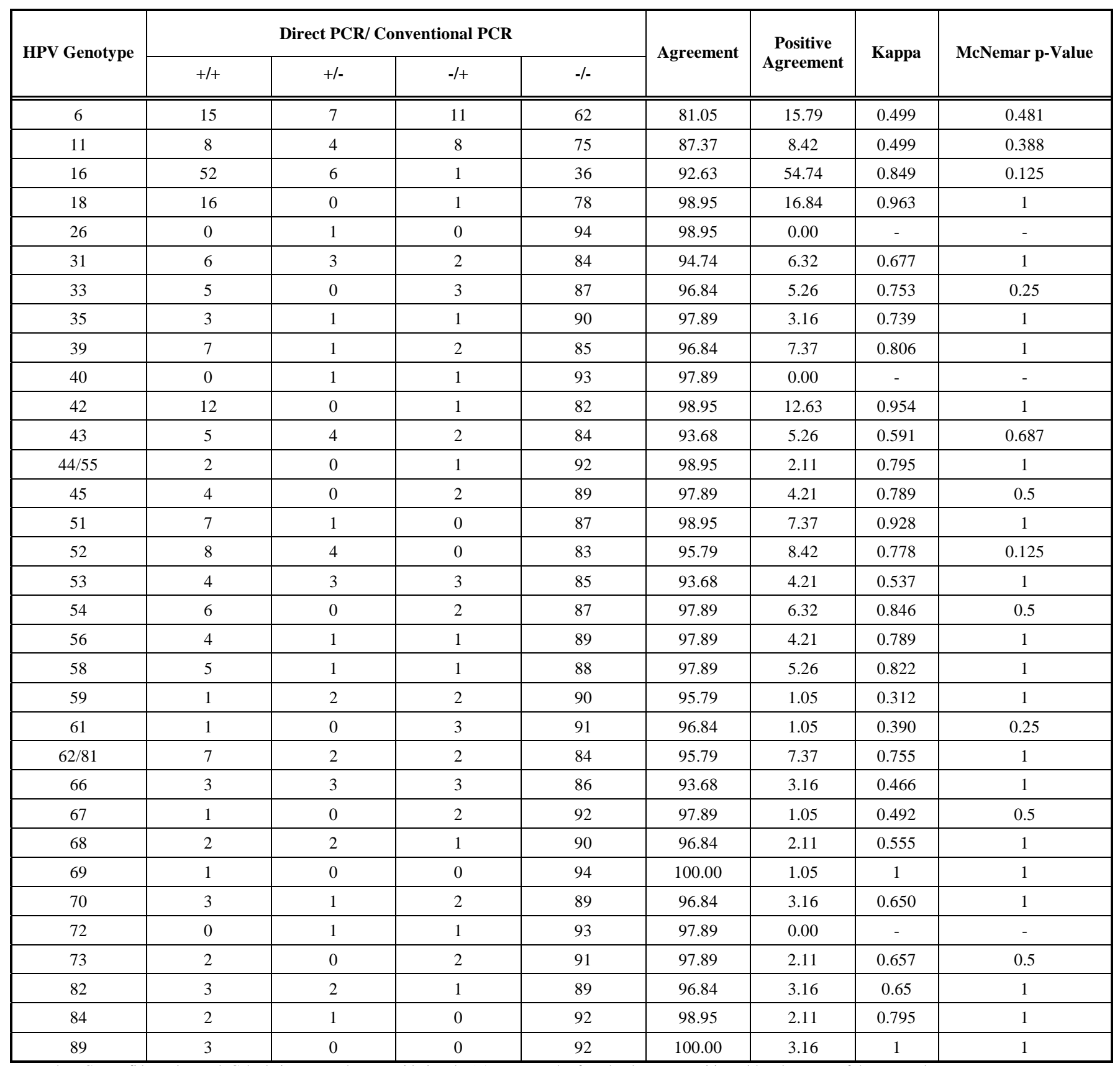

N: number. CI: confidence interval. Calculations were done considering the 95 HPV samples found to be HPV-positive with at least one of the protocols.

cervical lesions. The results from this study validate a new system that represents a powerful, simple and low-cost assay for HPV analysis in such samples.

\section{ABBREVIATIONS}

FFPE $=$ Formalin-fixed paraffin-embedded

CIN1 = CIN-grade 1 (cervical intraepithelial neoplasia)

CIN2 = CIN grade 2 (moderate dysplasia)

CIN3 = CIN grade 3 (severe dysplasia)

\section{CONFLICT OF INTEREST}

EHH, AO, and OP are employees of Master Diagnóstica.

\section{ACKNOWLEDGEMENTS}

The authors are grateful to Llenalia Garcia-Fernandez for the statistical analysis of the data and to Ana Olalla LopezGranados for technical assistance.

\section{REFERENCES}

[1] Walboomers JM, Jacobs MV, Manos MM, et al. Human papillomavirus is a necessary cause of invasive cervical cancer worldwide. J Pathol 1999; 189: 12-9.

[2] IARC. Monographs on Evaluation of Carcinogenic Risks of Humans. Human Papillomaviruses IARC 2005; 90.

[3] Wheeler CM, Kjaer SK, Sigurdsson K, et al. The impact of quadrivalent human papillomavirus (HPV; types 6, 11, 16, and 18) L1 virus-like particle vaccine on infection and disease due to 
oncogenic nonvaccine HPV types in sexually active women aged 16-26 years. J Infect Dis 2009; 199: 936-44.

[4] Paavonen J, Naud P, Salmeron J, et al. Efficacy of human papillomavirus (HPV)-16/18 AS04-adjuvanted vaccine against cervical infection and precancer caused by oncogenic HPV types (PATRICIA): final analysis of a double-blind, randomised study in young women. Lancet 2009; 374: 301-14.

[5] Gravitt PE, van Doorn LJ, Quint W, et al. Human papillomavirus (HPV) genotyping using paired exfoliated cervicovaginal cells and paraffin-embedded tissues to highlight difficulties in attributing HPV types to specific lesions. J Clin Microbiol 2007; 45: 3245-50.

[6] Baay MF, Quint WG, Koudstaal J, et al. Comprehensive study of several general and type-specific primer pairs for detection of human papillomavirus DNA by PCR in paraffin-embedded cervical carcinomas. J Clin Microbiol 1996; 34: 745-7.

[7] Herraez-Hernandez E, Alvarez-Perez M, Navarro-Bustos G, et al. HPV Direct Flow CHIP: A new human papillomavirus genotyping method based on direct PCR from crude-cell extracts. J Virol Methods 2013; 193: 9-17.
[8]

Quint WG, Scholte G, van Doorn LJ, Kleter B, Smits PH, Lindeman J. Comparative analysis of human papillomavirus infections in cervical scrapes and biopsy specimens by general SPF(10) PCR and HPV genotyping. J Pathol 2001; 194: 51-8.

[9] Perez C, Klaustermeier JE, Alemany L, Tous S, de Sanjose S, Velasco J. Comparison of 2 different PCR-based technologies for the detection of human papilloma virus from paraffin-embedded tissue: genomica clinical arrays versus $\operatorname{SPF}(10)-\mathrm{LiPA}(25)$. Diagn Mol Pathol 2012; 21: 45-52.

[10] Tan SE, Garland SM, Rumbold AR, Tabrizi SN. Human papillomavirus genotyping using archival vulval dysplastic or neoplastic biopsy tissues: comparison between the INNO-LiPA and linear array assays. J Clin Microbiol 2010; 48: 1458-60.

[11] Dona MG, Benevolo M, Pimpinelli F, et al. Comparative evaluation of different DNA extraction methods for HPV genotyping by linear array and INNO-LiPA. J Med Virol 2011; 83: 1042-7.

(C) Herraez-Hernandez, et al.; Licensee Bentham Open .

This is an open access article licensed under the terms of the Creative Commons Attribution Non-Commercial License (http: //creativecommons.org/licenses/by$\mathrm{nc} / 3.0 /$ ) which permits unrestricted, non-commercial use, distribution and reproduction in any medium, provided the work is properly cited. 